\title{
Research on Dynamic Modeling Of Virtual CNC System Of Human Machine Interface
}

\author{
BASHIR OSMAN \\ Department of Mechanical Engineering, Tianjin \\ University of Technology and Education, Tianjin, \\ 300134 \\ College of Information Engineering, Tianjin University \\ of Commerce, Tianjin, 300134, \\ Tianjin, China \\ Email: basheer931@hotmail.com
}

\author{
HU Deji \\ Department of Mechanical Engineering, Tianjin \\ University of Technology and Education, Tianjin, \\ 300134 \\ College of Information Engineering, Tianjin University \\ of Commerce, Tianjin, 300134, \\ Tianjin, China \\ Email: hudeji2000@yahoo.com.cn
}

\begin{abstract}
In this paper, a Virtual CNC system of Human machine interface Modeling described the structure of the real CNC system operator panel, virtual modeling and parser function of CNC panel using Delphi language programming logic as an advanced dynamic modeling concepts and techniques to carry out the virtual CNC system Human machine interface dynamic modeling in the trend to advance the overall level of the domestic NC simulation software industry.
\end{abstract}

Keywords- NC machining simulation; Virtual CNC system ; Man machine interface; secondary development; dynamic modeling.

\section{INTRODUCTION}

Now the virtual CNC simulation system is widely used in numerical control technology experiment teaching, metalworking, CNC operator training and production practice because of its benefits to save the investment, reduce supplies, save time and effort. Because the domestics software is commonly used the statics techniques and support limited types of tool model and NC machine software so more and more Virtual Machine tools are introduced and used in control software development and for realistic NC verification [1]. In such a Virtual Machine Tool 3 model representation for the simulation of the machine tool itself and machining model for simulation of the material removal at the work piece are combined [2,3]. The system is controlled by a software emulation of the numerical control. In this paper I will use the method of the analysis of the structure of the CNC tool panel, model establishment of virtual screen and virtual keyboard, , and writing a unified description of the file

\section{BASIC CONCEPT OF THE NC SYSTEM STRUCTURE AND DELPHI SOFTWARE}

Delphi is an object-oriented, visual programming environment for rapid application development. Delphi provides all the tools you need to develop, test, and deploy applications, including a large library of reusable components, a suite of design tools, application and form templates, and programming wizard [2]. Delphi come with with a component library made up of the objects, some of which are also components or control that used when writing code. The component library includes objects that are visual controls like datasets and timers. Objects descended from TComponent have properties and methods that allow them to be installed on the Component palette and added to Delphi forms and data modules. The components are highly encapsulated for example, buttons are programmed to respond to mouse clicks by firing OnClick events. If you use a button, you don't have to write code to handle generated events when the button is clicked. Most editions of Delphi come with a component library source code and examples of Delphi programming techniques [4].

The user interface contains the HMI software and its connector to the NC core as well as virtual control panel. This virtual control panel integrates the machine instrument panel and the operator panel into the one consistent user interface. The machine model is used to allow the visualization of the machine including the emulation and display of its Mechatronic behavior .ON the exiting CNC system panel screen modeling includes NC panel screen page, keyboard buttons, lights, and other components.

The control panel that allows a user/operator to communicate or talk to the machine and also provides feedback by displaying information that is relevant to the operator. This panel consists of a monitor, a keyboard and several other buttons [5]. The following picture fig. 1 shows FANUC Oi MD (milling machine) control panel structure group parts.

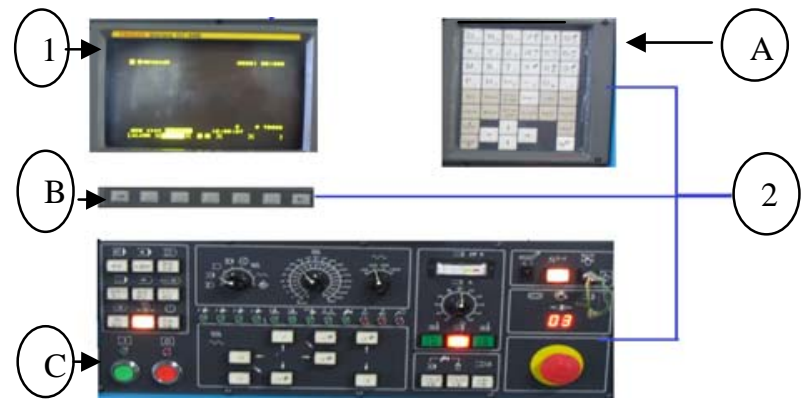

Fig. 1 Numercal Control Panel structure group 
1- Screen Display

2- Keyboard Panel contains: - A - Program panel B- Softkeybar C- HandelPnel.

\section{VIRTUAL NUMERICAL CONTROL PANEL MODELING}

According to the distribution of the actual numerical control system panel, dynamic modeling of the virtual panel includes Virtual Screen and Virtual Keyboard. The NC panel system (TNCControl) includes a CNC display screen (TNCControlScreen) and operation panel (TNCKeyPanel) . The following fig. 2 shows Screen dynamic modeling.
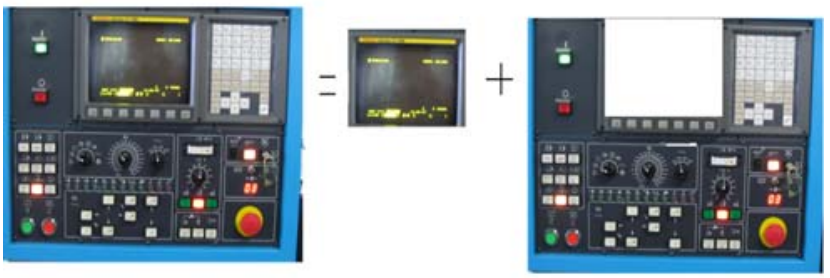

Fig. 2 Screen dynamic modeling.

\section{3-1 Display Screen Virtual Modeling}

As an example fig.3 FANUC Oi MD, CNC display screen consists of four parts : the title bar at the top, CNC screen page, status bar, and the menu bar on the button. Each of these parts has a basic CNC element such as NCLabel , NCText, NCMemo, TNClabelMemo, TNCFANUCParamMemo, and TNCLabelGrid.
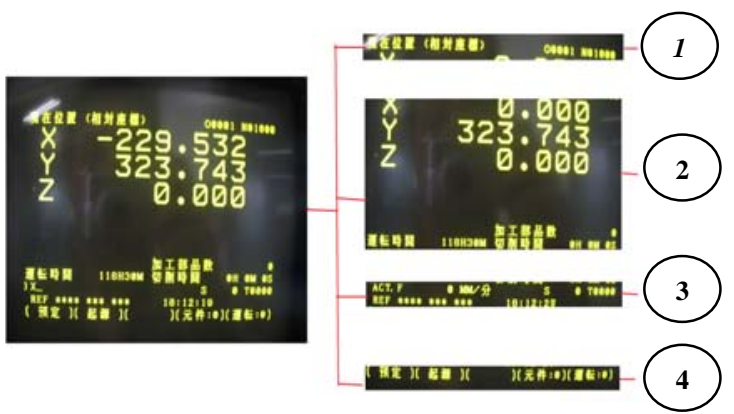

Fig. 3 Display screen modeling

1-The title bar 2- CNC screen page 3- The status bar 4- The menu bar.

The virtual display model is achieved through the establish of an object-oriented data model as follows: CNC display screen (TNCFAUCControlScreen) inherited from CNC display screen base class (TNCControlScreen), the FANUC display screen contains a main menu (TNCMenuItem), two status bar (TNCStatusBar) and an input buffer (TNCInputBuffer). FANUC CNC display screen contains multiple screen page group (TNCSreenGroup), each screen display page group include more than one screen display page (TNCScreenPage).

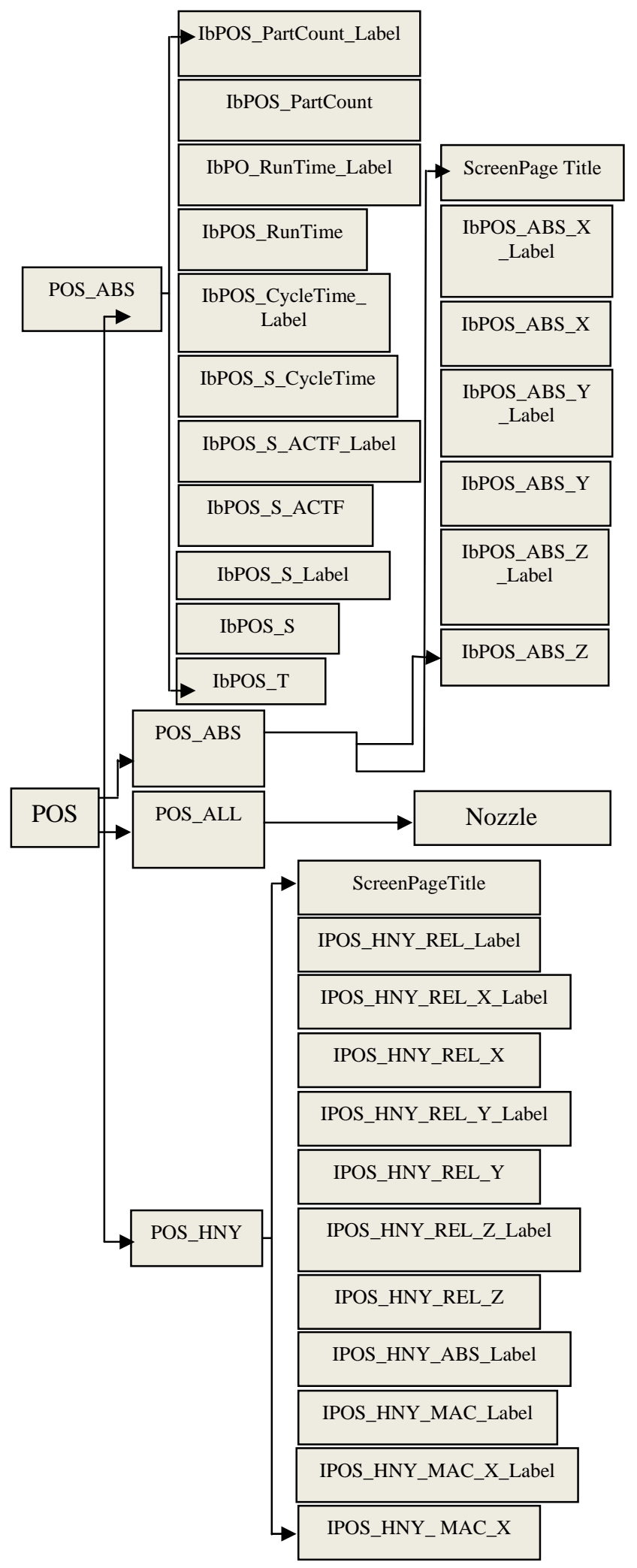

Fig. 4 POS Screen page group structure diagram 
(TNCSCreenPage), a screen display page containing multiple CNC basic element (TNCControlObej), the basic element derived basic screen display elements such as labels (TNCLabel), control Button (TNCButton), and (TNCLabelMemo) (6).

According to the POS function keys as fig.4 shows, page divided into for screen page group: POS_ABS , POS_ALL , and POS_HNY. Each screen page group sets up each object, which contains a description of each object's property, take POS_ABS as an example, object IbPOS_ABX_X label belonging to the component label which is used to output read-only text on the form " $X$ ", there is no built- in title used to mark control it contain the properties Left, Top, Width, height, AutoSize, Caption, Font. Color, Font. Height, Font. Name, Font. Style, and ParentFont used to indicate the position of the object, and font attributes. The object Label_ABX_X represented by the coordinate value of $\mathrm{X}$, it contains the properties Left, Top, Width, Height, AutoSize, Caption, alignment, Font. Color, Font. Height, Font. Name, Font. Style, and Parent. Font. The object mmPROG_MDI belongs to companies TNCMemo, the component TNCMemo can handle multiple lines of text.

The following example of a program fragment:

Lable sample: IbPOS_ABS_X Label: TLabel \{

$$
\begin{aligned}
& \text { Left }=40 \\
& \text { Top }=0 \\
& \text { Width }=40 \\
& \text { Height }=80 \\
& \text { AutoSize = False } \\
& \text { Caption = "X" } \\
& \text { Font. Color = CIYellow } \\
& \text { Font. Height }=16 \\
& \text { Font. Name = "MSsansSerif" } \\
& \text { ParentFont }=\text { False } \\
& \text { Font. Height = } 16 \\
& \text { Font. Name }=\text { "Fixedsys" } \\
& \text { TNCMemo }\{ \\
& \text { Left } 10 \\
& \text { Top } 0 \\
& \text { WEIGHT } 350 \\
& \text { VISABLE TRUE } \\
& \text { Color = C Yello } \\
& \text { Font. Height = } 16 \\
& \text { Font. Name = "Fixedys" }
\end{aligned}
$$

Memo as an example of the red piece: mmPROG_MDI; TNCMemo \{

\section{V .MODELING OF VIRTUAL KEYBOARD}

With FANUC Oi NC SYSTEM as an example, dynamic modeling of virtual CNC keyboard includes virtual system keyboard modeling, virtual soft keyboard modeling, fig.5 shows various parts of the keyboard : turn, button, lights, switches, hand wheel, start and stop switch components. To simplify the model as described, I will abstract lights, hand wheel, start-stop switch and other components to express the button.

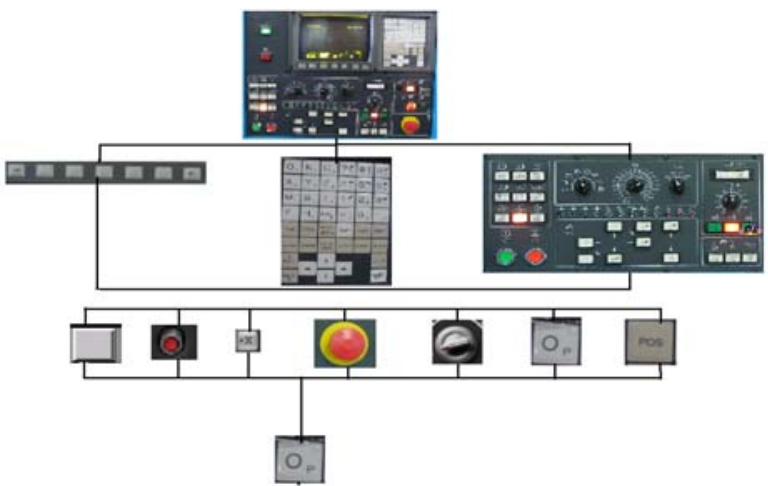

Fig.5 keyboard modeling

An operation panel contains more than one group of buttons (TNCButtonGroup), a button group contains more than one button (TNcButton). Fig. 6 shows an a keyboard diagram.

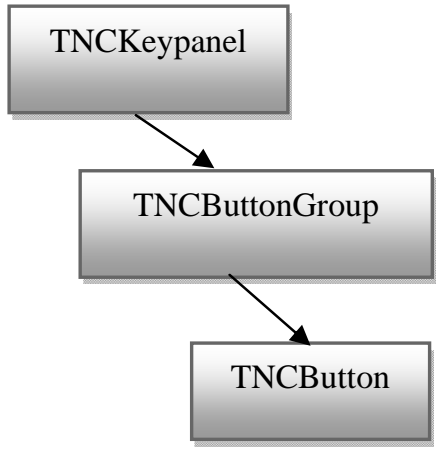

Fig.6 keyboard diagram

The soft keyboard is set to a button group BITMAP_ BUTTON_GROUP , The softkey button

Defines to seven buttons: : SOFT_KEY_RETURN, SOFT_KEY_1 , SOFT_KEY_2 , SOFT_KEY_3 , SOFT_KEY_4, SOFT_KEY_5, SOFT_KEY_NEXT Each button with BUTTON_STYLE, BUTTON_POSITION_RECT , KEY_TYPE

KEY_VALUE , STATUS_BMP_PATH . Figure 2-14 shows operator panel structure diagram :-

Machine Status Group) Contains 9 buttons components : SINGLE_STEP , RUN_TEST , SINGLE_STEP_INGORE , OPTINALL_STOP, MACHI_LOCK , MST_LOCK , ZAXIS_LOCK , DOOR_LOCK, and SYSTEM START.

(Machine Status Led Group) contains 12 buttons components : XAXIS_ZERO , YAXIX_ZERO , ZAXIX_ZERO , AAXIX_ZERO , XAXIX_MIROR , YAXIX_MIROR , ATC_ZERO, FIXTURE , LIQUID , NC_START, MC_START, and AIR . 
NC Mode Switch Group) include (MODE_SWICH), (FEED SWITCH) , and (RABID_SWITCH).

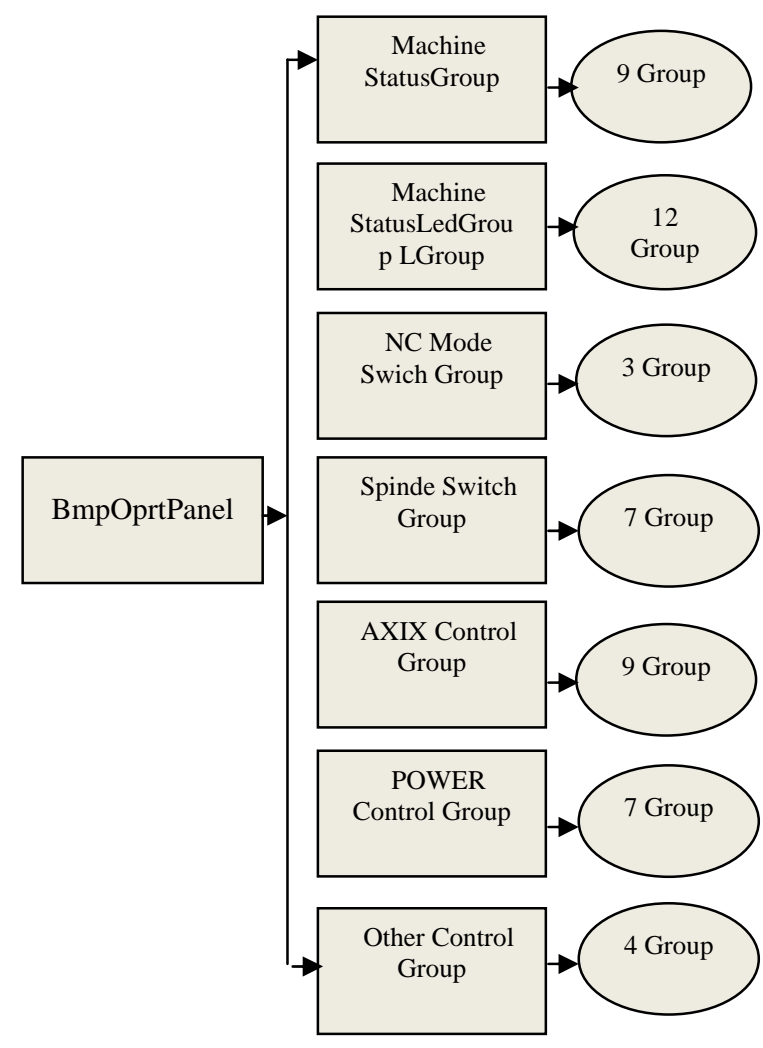

Fig.7 Operation Panel Structure

(SPINDE SWITCH GROUP) includes 7button : SPINDE CW , SPINDE STOP , SPINDLE CCW , CUTTING_LIQUID_AUTO, CUTTING_LIQUID_MANU , and SPINDLE BLOW.

(AXIXS CONTROL GROUP) includes 9 buttons: $\mathrm{X}$ forward direction (XAXIS_POSITIVE) , A negative (AAXIS_NEGATIVE) , Y positive (YAXIS_POSITIVE) , Y negative (Y AXIS_NEGATIVE) , A positive (AAXIS_POSITIVE), X negative (XAXIS_NEGATIVE), $Z$ positive (ZAXIS-POSITIVE) , $Z$ positive (ZAXIS_POSITIVE) , Z negative (ZAXIS_NEGATIVE), HANDEL_BTN.

(POWER Control Group) includes 7 buttons : SYCLE_START_LED , CYCLE_STOP_ LED CYCLE_START , CYCLE_STOP , POWR ON , POWER OF , EMERGENCY_BUTTON.
(OTHER CONTROL GROUP) IS DEFINITION OF THE FOUR BUTTONS: (SPINDLE_SWICH), (FEEDING_SWICH) , (H_SWICH), (SPINDLE_LOCK).

System panel described as a bitmap button group : as follows:-

1- (BITMAP_BUTTON_GROUP) defines 44 BITMAP button .Such as OP, NQ , GR , 7A , 8B , 9D , POS , PROG And other keyboard button

2- - Some examples of the state of the machine group: - BITMAP_BUTTON_GROUP "Machine Status Group” \{

KEYPAMEL_KEY_COUNT 9

BITMAP_BUTTON “SINGLE_STEP” \{

BUTTON_STYLE bsSwitch

BUTTON_POSITION_RECT $(41,42,30,30)$ \}

BITMAP_BUTTONSINGLE_STEP_INGORE” \{ BUTTON_STYLE bsSwitch

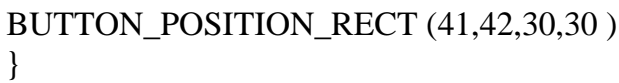

\section{CONCLUSION}

Dynamic modeling of virtual human machine interface technology aims to solve shortage of the domestic CNC simulation software and add a secondary development interface function for NC machining simulation replacement and updating asked of multi systems. Another side is to facilitate teaching and training for the student and save money by solving the problem of current NC machine simulation.

\section{REFERENCES}

[1] Hohwieler, E. Internet-Zugang an Steuerungen für

Produktionsunterstützung und Teleservice. In: Krause, F.-L. And Uhlmann, E. (Ed.) Innovative Produktionstechnik. Hanser, München, 1998, pp.505518

[2] Bärnklau, A. Mehr als CNC-Ausbildung. Spektrum SinuTrain, 3. Jahrgang, März 2003, Pp. 10-11

[3] Blöchl, W. And Bärnklau, A. And Schröder, T. Ausbildung wird zum Wettbewerbsvorteil. NCFertigung 6-01, 2001, pp. 114-116

[4] Borland ${ }^{\circledR}$ Delphi $^{\mathrm{TM}} 7$ for Windows ${ }^{\mathrm{TM}}$. Borland International, Inc., 100 Borland Way P.O. Box 660001, Scotts Valley, CA 950670001www.borland.com

[5] USING HFE TO DESIGN A USER-FRIENDLY CNC CONTROL PANEL, Presented to Dr. Clauson, Term Paper QAS 515.

[6] DYAAMIC MODELING TECHNOLOGY OF-MAN MACHINE INTERFACE OF VIRTUAL CNC SYSTEM.

M.S.DISSERTATION 2010. PP. 7-2O 